\title{
Analyse des évaluations de dose utérine réalisées par l'IRSN de 2004 à 2008
}

\author{
C. ÉTARD ${ }^{1}$, B. AUBERT ${ }^{1}$
}

(Manuscrit reçu le 16 juillet 2009, accepté le 24 août 2009)

RÉSUMÉ Suite à l'exposition médicale le plus souvent fortuite, d'une patiente enceinte, le praticien peut solliciter l'IRSN pour évaluer la dose délivrée au foetus. De 2004 à 2008, 326 doses utérines ont été évaluées dans ce contexte par l'IRSN, consécutivement à des examens de radiologie ou de médecine nucléaire, dont 246 correspondaient à des situations où l'utérus était directement exposé. Les évaluations dosimétriques ont été réalisées par calcul sur la base des paramètres techniques de l'examen. En radiologie conventionnelle, les doses utérines évaluées sont inférieures à $35 \mathrm{mGy}$ et leurs moyennes en accord avec les données internationales. En scanographie, les doses sont généralement comprises entre 15 et $65 \mathrm{mGy}$ mais peuvent dépasser dans certaines configurations la valeur de $100 \mathrm{mGy}$. Dans ce domaine, une attention particulière doit donc être apportée à l'optimisation des examens du pelvis chez la femme et une évaluation individuelle de la dose délivrée doit être réalisée si la grossesse est avérée. Par ailleurs la dispersion des doses pour un même type d'examen radiologique reflète des pratiques hétérogènes, parfois éloignées des recommandations de la Société française de radiologie. En médecine nucléaire, les doses utérines évaluées sont inférieures à $10 \mathrm{mGy}$, sans tenir compte de la dose liée à un éventuel examen scanographique associé.

ABSTRACT Analysis of foetal dose assessed by IRSN from 2004 to 2008.

After a medical exposure of a pregnant patient, the practitioner can seek IRSN to estimate the foetal dose. From 2004 to 2008,326 foetal doses have been estimated by IRSN following radiological or nuclear medicine examinations, among them 246 corresponded to situations where uterus was directly exposed. These estimations were performed by calculations based on the technical parameters of the exam. In conventional radiology, foetal doses estimated are lower than $35 \mathrm{mGy}$ and their averages are in agreement with international data. In computed tomography, doses are generally in the range 15 to $65 \mathrm{mGy}$, but can exceed $100 \mathrm{mGy}$ in certain configurations. In this field, practitioners must pay a particular attention to the optimization of pelvic exams of female patients and estimate individually the foetal dose in case of pregnancy. Furthermore, the dispersal of doses for the same type of radiological exam shows heterogeneous practices, sometimes very far from the recommendations of the French society of radiology. In nuclear medicine, foetal doses estimated are lower than $10 \mathrm{mGy}$, without taking into account the dose due to a possible associated CT examination.

Keywords: medical exposure / fetal dose / radiology / computed tomography / nuclear medicine

\footnotetext{
1 IRSN, Unité d'expertise en radioprotection médicale, DRPH/SER/UEM, BP 17, 92262 Fontenay-aux-Roses Cedex, France.
} 


\section{Introduction}

La nouvelle réglementation relative à la radioprotection des patients a sensibilisé les médecins, et les patients eux-mêmes, sur le risque lié à l'exposition aux rayonnements ionisants in utero.

À la suite de l'exposition d'une femme enceinte, le radiologue, ou le médecin nucléaire, est de plus en plus fréquemment interrogé, par la patiente ou son médecin traitant, sur la dose qui a été reçue par l'embryon lors de l'examen.

Afin d'évaluer cette dose, l'Institut de radioprotection de sûreté nucléaire (IRSN) est régulièrement sollicité par les praticiens. Ainsi, de 2004 à 2008, 326 évaluations de dose utérine ont été réalisées par l'IRSN suite à l'exposition, le plus souvent fortuite, d'une femme enceinte à des fins de diagnostic médical. Cet article présente un bilan de ces évaluations.

\section{Matériel et méthodes}

\subsection{Répartition des demandes selon le domaine d'activité}

Le principal domaine concerné par les évaluations de dose utérine réalisées par l'IRSN est la radiologie (conventionnelle ou scanographie) avec 307 cas sur 326. Parmi ces 326 cas, 246 correspondent à des situations où l'utérus est directement exposé, soit environ $75 \%$ des demandes reçues (Tab. I).

La figure 1 présente la répartition des demandes selon le type d'acte dont avait bénéficié la patiente: radiologie seule, scanographie seule, radiologie et scanographie, ou médecine nucléaire, en différenciant les cas où le fœus était ou non directement exposé.

\section{TABLEAU I}

Évolution du nombre d'évaluations de doses utérines demandées à l'IRSN de 2004 à 2008, suite à une exposition à des fins de diagnostic médical.

Evolution of the number of foetal dose estimations performed by IRSN after medical exposure, from 2004 to 2008 .

\begin{tabular}{lcccccc}
\hline & $\mathbf{2 0 0 4}$ & $\mathbf{2 0 0 5}$ & $\mathbf{2 0 0 6}$ & $\mathbf{2 0 0 7}$ & $\mathbf{2 0 0 8}$ & Total 2004-2008 \\
\hline Nombre d'évaluations & 45 & 50 & 57 & 87 & 87 & 326 \\
$\begin{array}{l}\text { avec utérus dans le champ } \\
\text { ou médecine nucléaire }\end{array}$ & $\mathbf{3 2}$ & $\mathbf{4 1}$ & $\mathbf{4 2}$ & $\mathbf{6 6}$ & $\mathbf{6 5}$ & $\mathbf{2 4 6}$ \\
\hline
\end{tabular}




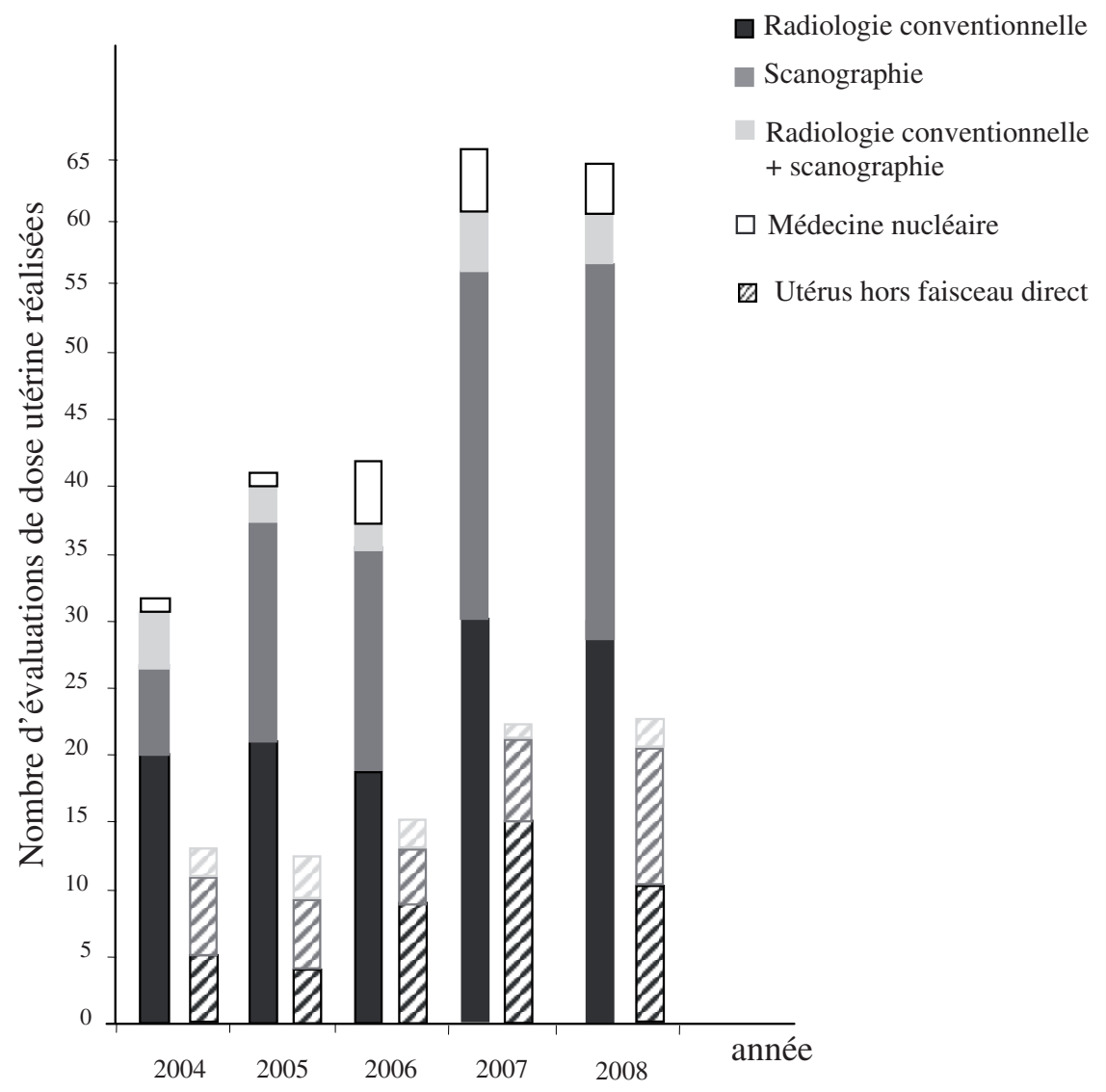

Figure 1 - Répartition des évaluations de dose utérine réalisées entre 2004 et 2008, selon le type d'acte en précisant si le fretus est, ou non, directement exposé.

Distribution by image modality of foetal dose estimations performed between 2004 and 2008, with uterus directly or indirectly exposed.

\subsection{Méthodes de calcul de dose}

Les évaluations de dose utérine ont été réalisées sur la base des paramètres techniques de l'examen, transmis par le médecin ayant réalisé l'acte et à l'aide de moyens de calcul adaptés au domaine d'activité.

\subsubsection{En radiologie conventionnelle}

Pour permettre l'évaluation de la dose utérine reçue par sa patiente, le médecin ayant réalisé l'acte doit transmettre à l'IRSN, via un formulaire type, les 
principaux paramètres techniques qui influent directement sur la dose reçue au niveau de l'utérus :

- le nombre de radiographies réalisées, leurs dimensions et incidences ;

- pour chacune de ces radiographies : la haute tension et la charge électrique ;

- le cas échéant, le temps et le courant de scopie ;

- la filtration.

Le poids et la taille de la patiente sont également nécessaires à l'évaluation de la dose utérine.

Dans la plupart des cas, la grossesse était inconnue de la patiente au moment de l'examen. Le recueil des paramètres doit être effectué a posteriori, alors que l'intégralité de ces paramètres n'est plus disponible sur l'installation. C'est en particulier le cas de la charge électrique pour les installations de radiologie fonctionnant avec un exposeur automatique (ou cellule), ou du temps de scopie que les utilisateurs doivent « apprécier » parfois plusieurs jours après la réalisation de l'acte... Dans le cas où certaines données sont inconnues, l'évaluation est réalisée sur la base des paramètres recommandés dans les procédures établies par la Société française de radiologie (SFR) et l'IRSN ${ }^{2}$.

Jusqu'en septembre 2006, les doses ont été calculées manuellement sur la base des paramètres techniques de l'examen cités ci-dessus. Depuis cette date, les évaluations sont réalisées grâce au logiciel «PCXMC» V1.5.2 puis V2.0 (PCXMC, 2008), qui utilise un code de calcul type Monte Carlo pour calculer les doses délivrées aux organes en radiologie conventionnelle. Ce logiciel tient compte de la morphologie de la patiente (taille et poids). Les expositions étudiées ayant eu lieu pour la plupart dans les premières semaines de la grossesse, la dose à l'utérus est considérée comme représentative de la dose au fœtus.

\subsubsection{En scanographie}

Les paramètres techniques de l'examen nécessaires à l'évaluation de la dose utérine en scanographie sont les suivants :

- la marque et le type de scanner ;

- la région anatomique explorée ;

- le nombre d'acquisitions réalisées, leur longueur et leur positionnement ;

- pour chacune de ces acquisitions : la haute tension, la charge électrique par rotation, le pitch $^{3}$, l'index dosimétrique (CTDIvol) et le produit dose $\times$ longueur (PDL) ;

- le nombre de détecteurs et la largeur de collimation.

\footnotetext{
2 Les procédures radiologiques : réalisation, critères de qualité et optimisation. Société française de radiologie et Institut de radioprotection et de sûreté nucléaire, http://www.sfrnet.org ou lien direct: http://pagesperso-orange.fr/eassa.cordo/ SFROPRI/index.htm

3 Le pitch est le rapport entre le déplacement de table par rotation de $360^{\circ}$ et la largeur de collimation.
} 
Le poids et la taille de la patiente sont également nécessaires à l'évaluation de la dose utérine. Si certains paramètres sont inconnus, ils sont estimés à partir des recommandations du guide des procédures SFR/IRSN.

Jusqu'en septembre 2006, la dose reçue à l'utérus était calculée manuellement sur la base des paramètres techniques de l'examen. Cette méthode «manuelle » d'évaluation de dose utérine, qui assimile le pelvis de la patiente à un cylindre de plexiglas de $32 \mathrm{~cm}$ de diamètre et considère l'utérus sur l'axe de ce cylindre, conduit à estimer la dose utérine égale à CTDIvol x 3/5.

Depuis cette date, les évaluations sont réalisées grâce au logiciel «CTExpo » V1.5 puis V1.6, qui utilise la méthode de Monte Carlo pour calculer les doses délivrées aux organes en scanographie, pour une patiente de morphologie standard.

L'écart systématique relevé entre ces deux méthodes de calcul a conduit l'IRSN à réaliser une étude dosimétrique sur fantôme anthropomorphe (IRSN, 2009). Cette étude a montré que la méthode de calcul manuelle employée de 2004 à septembre 2006 sous-estimait la dose à l'utérus d'un facteur 1,8 à 2,3 selon le type de scanner et la position de l'utérus par rapport à la limite inférieure de la zone explorée. Ces résultats confirment ceux de Wagner et al. (1997).

Pour l'étude objet de cet article, toutes les évaluations dosimétriques réalisées entre 2004 et 2006 suite à des examens scanographiques ont été reprises, à l'aide du logiciel CTExpo.

\subsubsection{En médecine nucléaire}

Les doses délivrées aux organes sont directement liées au type de radiopharmaceutique utilisé et proportionnelles à l'activité administrée.

Les évaluations de dose utérine sont réalisées en s'appuyant sur le rapport de la Société française de physique médicale n¹9-2001 (SFPM, 2001) « Dosimétrie des explorations diagnostiques en médecine nucléaire », basé sur les données des rapports $n^{\circ} 53$ et $n^{\circ} 80$ (ICRP, 1987, 1998) de la Commission internationale de protection radiologique (CIPR).

\section{Résultats et analyse}

Seuls les actes de radiodiagnostic au cours desquels l'utérus se trouve dans ou à proximité du faisceau direct et les examens de médecine nucléaire peuvent induire une dose utérine significative (supérieure à $0,2 \mathrm{mGy}$ ). Les résultats présentés portent exclusivement sur ces types d'actes, soit 246 cas ( $\approx 75 \%$ des demandes). 
Cette valeur « seuil » choisie égale à 0,2 mGy se justifie au regard de l'exposition maximale réglementaire autorisée pour le fœtus, égale à $1 \mathrm{mSv}$ (ou $1 \mathrm{mGy}$ sur le fœetus entier).

Parmi les 246 évaluations de dose utérine réalisées, 110 faisaient suite à un examen scanographique, 18 d'entre eux étant associés à un examen de radiologie conventionnelle et 2 à un examen TEP 4 .

Les résultats des évaluations sont présentés successivement pour les différents domaines : radiologie conventionnelle, scanographie et médecine nucléaire. Pour les patientes ayant bénéficié à la fois d'un examen radiologique et d'un examen scanographique, les évaluations des deux composantes sont analysées indépendamment. Les résultats globaux, présentés au paragraphe 4, distinguent les patientes dans ce cas.

\subsection{En radiologie conventionnelle}

Parmi les 246 évaluations de dose utérine réalisées, 138 faisaient suite à un examen de radiologie conventionnelle, 18 étant associés à un examen scanographique. La figure 2 présente la répartition de ces examens. Les valeurs de dose utérine

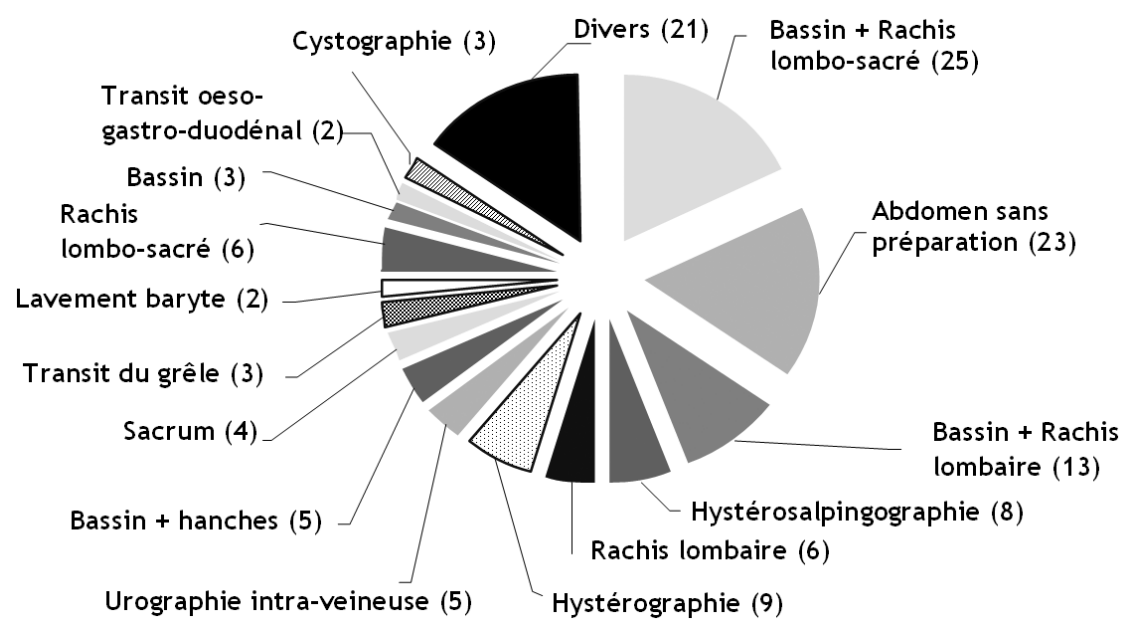

Figure 2-Répartition des types d'examen de radiologie conventionnelle ayant fait l'objet d'une évaluation de dose utérine.

Distribution of the assessed examinations by anatomical areas, in conventional radiology.

\footnotetext{
4 Tomographie par émission de positons.
} 


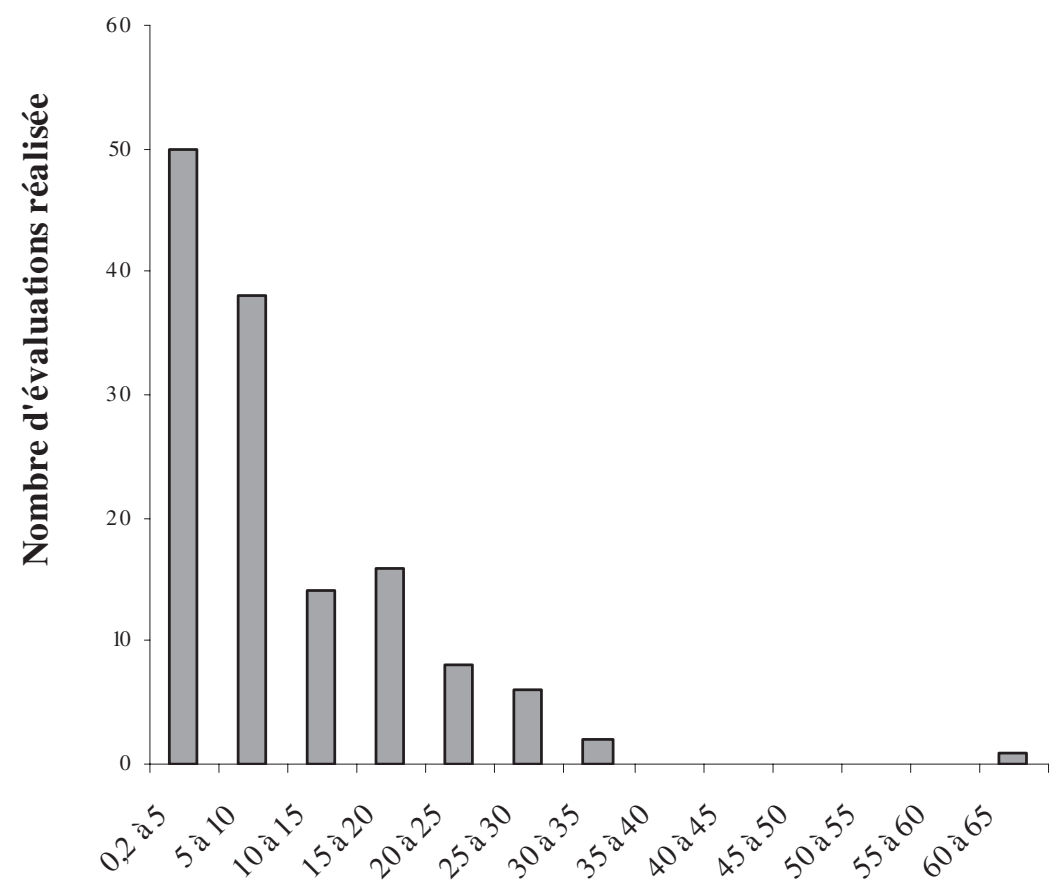

Dose utérine (mGy)

Figure 3 - Distribution des doses utérines évaluées relatives à des examens de radiologie conventionnelle (utérus dans ou à proximité du faisceau direct).

Distribution of the estimated foetal doses after radiological examinations in conventional radiology (uterus in or close to the direct beam).

évaluées selon la méthode décrite au paragraphe 2.2.1 s'étendent de 0,2 mGy à $35 \mathrm{mGy}$, selon la distribution présentée sur la figure 3, à l'exception d'une hystérographie qui a conduit à une dose de 64 mGy.

Pour les 2 examens les plus représentés, abdomen sans préparation (ASP) et bassin + rachis lombo-sacré (RLS), les valeurs de dose utérine moyennes et médianes, ainsi que les valeurs minimales et maximales, sont présentées dans les tableaux II et III. Ces valeurs correspondent à l'examen complet, radiographies et radioscopie éventuelle. Elles sont comparées aux données de la littérature internationale.

L'écart relevé entre les doses utérines minimale et maximale est très important pour un même type d'examen : elles varient d'un facteur 12 pour le bassin + RLS et 36 pour l'ASP. Ces facteurs s'expliquent essentiellement par le nombre de 


\section{TABLEAU II}

Doses utérines pour l'examen radiologique complet de l'ASP (doses moyenne, médiane, minimale et maximale). Comparaison aux données internationales.

Foetal dose following abdomen examination (mean, median, minimum and maximum doses). Comparison with international data.

\begin{tabular}{|c|c|c|c|}
\hline & $\mathrm{D}_{\mathrm{moy}}(\mathrm{mGy})$ & $\mathrm{D}_{\text {med }}(\mathrm{mGy})$ & $D_{\text {mini }}-D_{\operatorname{maxi}}(m G y)$ \\
\hline Cette étude & 2,6 & 2,5 & $0,2-7,2$ \\
\hline Données internationales & $1,4^{\mathrm{c}}$ & $\begin{array}{l}1,8^{\mathrm{a}} \\
2,9^{\mathrm{b}}\end{array}$ & $\begin{array}{c}0,25-18,3^{\mathrm{a}} \\
0,28-15^{\mathrm{b}} \\
\text { na }-4,2^{\mathrm{c}}\end{array}$ \\
\hline
\end{tabular}

\section{TABLEAU III}

Doses utérines pour l'examen radiologique complet du bassin + RLS (doses moyenne, médiane, minimale et maximale). Comparaison aux données internationales.

Foetal dose following "pelvis + lumbar spine +sacrum" examination (mean, median, minimum and maximum doses). Comparison with international data.

\begin{tabular}{cccc|}
\hline & $\mathbf{D}_{\text {moy }}(\mathbf{m G y})$ & $\mathbf{D}_{\text {med }}(\mathbf{m G y})$ & $\mathbf{D}_{\text {mini }}-\mathbf{D}_{\text {maxi }}(\mathbf{m G y})$ \\
\hline Cette étude & $\mathbf{9 , 6}$ & $\mathbf{7 , 9}$ & $1,9-23,3$ \\
Données internationales & $\mathbf{4 , 8}^{\mathrm{a}}$ & $\mathbf{4 , 0}^{\mathrm{a}}$ & $0,6-19,6^{\mathrm{a}}$ \\
& $\mathbf{1 2 , 9}^{\mathrm{b}}$ & & $1,9-60^{\mathrm{b}}$ \\
\hline
\end{tabular}

${ }^{\mathrm{a}}$ NRPB-R200 (1986) - Données correspondant à l'examen du bassin + rachis lombaire ; ${ }^{\mathrm{b}}$ données adaptées de Osei et Faulkner (1999).

radiographies réalisées (respectivement 3 à 9 et 1 à 3 ), la variabilité des paramètres physiques de l'examen (particulièrement la haute tension et la charge) et par le temps d'utilisation de la scopie. Rappelons que la morphologie de la patiente, qui influe sur le choix des paramètres techniques de l'examen, est prise en compte par le logiciel « $\mathrm{PCXMC} »$. Ils illustrent également le fait que les conditions de réalisation de certains examens s'écartent des recommandations des procédures de la SFR.

On constate cependant que les doses utérines moyennes évaluées dans notre étude, ainsi que leur dispersion pour à un ASP ou un examen du bassin + RLS sont comparables à celles de la littérature.

\subsection{En scanographie}

La figure 4 présente la répartition des 110 examens de scanographie selon le type d'examen.

Les valeurs de dose utérine évaluées selon la méthode décrite au paragraphe 1.2.2 s'étendent de $2 \mathrm{mGy}$ à $127 \mathrm{mGy}$, selon la distribution présentée sur la figure 5 . 
Foie (2)

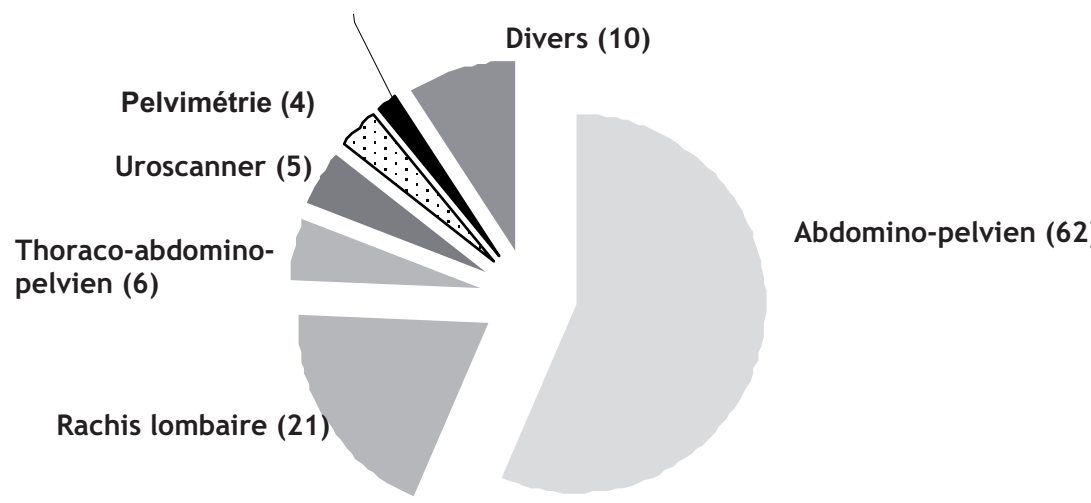

Figure 4 - Répartition des types d'examen de scanographie ayant fait l'objet d'une évaluation de dose utérine.

Distribution of the assessed examinations by anatomical areas, in computed tomography.

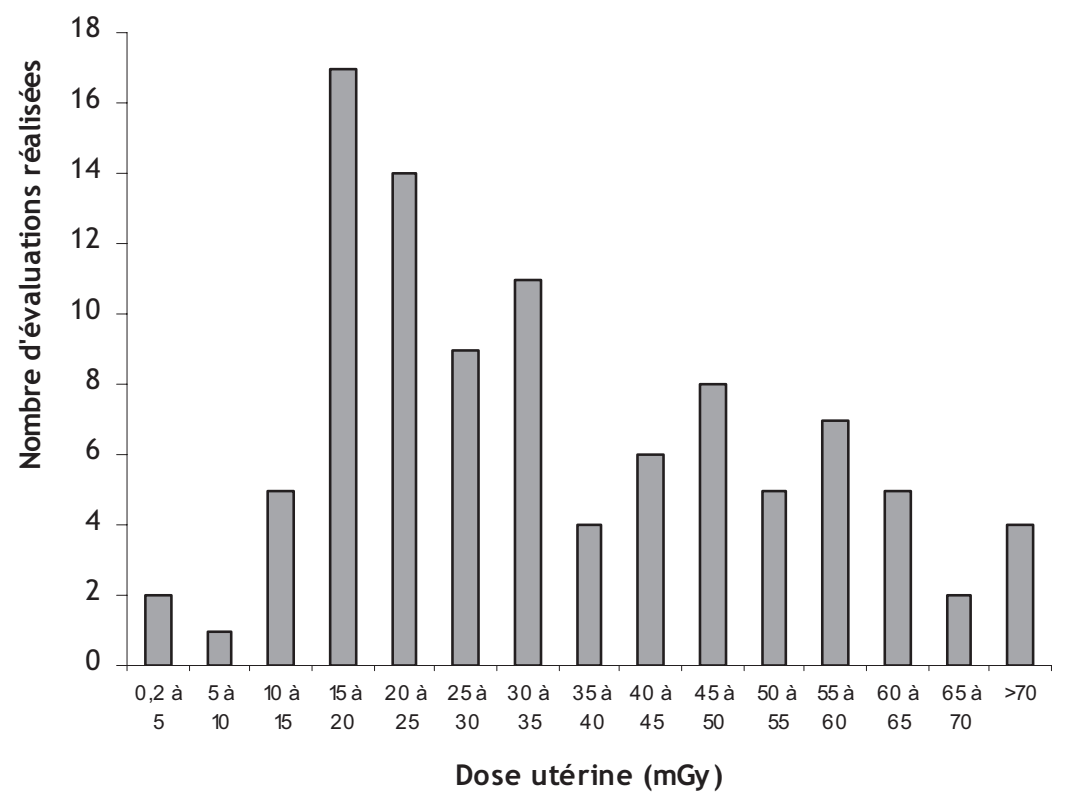

Figure 5 - Distribution des doses utérines évaluées relatives à des examens de scanographie.

Distribution of the estimated foetal doses after CT examinations (uterus in or close to the explored area). 
TABLEAU IV

Doses utérines pour l'examen scanographique abdomino-pelvien (doses moyenne, médiane, minimale et maximale). Comparaison aux données internationales.

Foetal dose following “abdomen + pelvis" CT examination (mean, median, minimum and maximum doses). Comparison with international data.

\begin{tabular}{cccc|} 
& $\mathbf{D}_{\text {moy }}(\mathbf{m G y})$ & $\mathbf{D}_{\text {med }}(\mathbf{m G y})$ & $\mathbf{D}_{\text {mini }}-\mathbf{D}_{\text {maxi }}(\mathbf{m G y})$ \\
\hline Cette étude & $\mathbf{3 6}$ & $\mathbf{3 0}$ & $12-127$ \\
Données internationales & $\mathbf{2 5}^{\mathbf{a}}$ & & $\mathrm{na}-79^{\mathrm{a}}$ \\
\hline a Sharp et al. (1998). & &
\end{tabular}

\section{TABLEAU V}

Doses utérines pour l'examen scanographique du rachis lombaire (doses moyenne, médiane, minimale et maximale). Comparaison aux données internationales.

Foetal dose following lumbar spine CT examination (mean, median, minimum and maximum doses). Comparison with international data.

\begin{tabular}{cccc}
\hline & $\mathbf{D}_{\text {moy }}(\mathbf{m G y})$ & $\mathbf{D}_{\text {med }}(\mathbf{m G y})$ & $\mathbf{D}_{\text {mini }}-\mathbf{D}_{\text {maxi }}(\mathbf{m G y})$ \\
\hline Cette étude & $\mathbf{4 0}$ & $\mathbf{4 2 , 5}$ & $5,4-66$ \\
Données internationales & $\mathbf{2 , 4 ^ { \mathrm { a } }}$ & & $\mathrm{na}-8,6^{\mathrm{a}}$ \\
\hline
\end{tabular}

a Sharp et al. (1998).

Les évaluations ont été réalisées sur la base d'une patiente de morphologie standard $(1,60 \mathrm{~m}$ et $60 \mathrm{~kg})$ qui est celle retenue par le logiciel CTExpo. Elles surestiment donc la dose réellement reçue pour les patientes plus fortes et sous estiment la dose dans le cas de patientes plus minces.

Pour les 2 examens les plus représentés (abdomino-pelvien et rachis lombaire), les valeurs de dose utérine moyennes et médianes, ainsi que les valeurs minimales et maximales, sont présentées dans les tableaux IV et $\mathrm{V}$. Ces valeurs correspondent à l'examen complet (une ou plusieurs acquisitions). Elles sont comparées aux données de la littérature internationale.

L'écart relevé entre les doses utérines minimale et maximale est très important pour un même type d'examen (facteur 10 environ).

Pour l'examen abdomino-pelvien, cet écart s'explique essentiellement par le nombre d'acquisitions réalisées (entre 1 et 3 selon les actes analysés), et surtout en raison de la grande variabilité de l'index dosimétrique (CTDIvol), parfois très éloigné des recommandations de la SFR.

Dans le cas de l'examen du rachis lombaire, chaque acte analysé ne comportait qu'une acquisition. L'important écart de dose est donc uniquement lié à l'utilisation de paramètres techniques très variables, et là aussi parfois très éloignés des recommandations de la SFR. 
La marque et le type de scanner sont également à prendre en considération. En effet, à paramètres d'acquisition équivalents, tous ne délivrent pas la même dose au patient. En conséquence, la dose utérine pour ces deux types d'examen scanographique peut varier d'un facteur 10 selon la procédure utilisée et le type de scanner et atteindre, si plusieurs acquisitions sont réalisées, des valeurs égales ou supérieures à 100 mGy.

Même si les calculs réalisés dans cette étude ne prennent pas en compte la morphologie de la patiente, ils sont en bon accord avec les données de Sharp pour l'examen abdomino-pelvien mais très supérieurs pour l'examen du rachis lombaire. Il est à noter que, conformément aux indications des praticiens, les évaluations de dose utérines suite à des examens scanographiques du rachis lombaire ont systématiquement été réalisées en considérant l'utérus à l'intérieur de la zone explorée. La faible valeur de dose publiée par Sharp laisse à penser que son étude a été réalisée en considérant l'utérus dans le rayonnement diffusé.

Compte tenu des niveaux de dose et de leur dispersion, il est indispensable pour les examens scanographiques au cours desquels l'utérus se trouve dans la zone explorée d'évaluer la dose utérine délivrée avec les paramètres propres à l'examen plutôt que de se rapporter aux valeurs moyennes disponibles dans la littérature, et de positionner convenablement l'utérus par rapport à la zone explorée, selon les indications du praticien.

\subsection{En médecine nucléaire}

Parmi les 19 évaluations de dose utérine suite à un examen de médecine nucléaire, 2 étaient des examens TEP associés à un examen scanographique (TEP-TDM).

La répartition des types d'examens et les estimations de dose utérine associées à ces examens sont présentées respectivement sur les figures 6 et 7 .

Il convient de noter que les deux examens de TEP-SCAN étudiés conduisent à des doses utérines respectivement de 17 et $27 \mathrm{mGy}$. La contribution dosimétrique de l'examen scintigraphique au fluor-18 était estimée dans les deux cas à 7 mGy.

Les examens étudiés induisent donc tous des doses utérines inférieures ou égales à 10 mGy, auxquelles il convient d'ajouter la dose éventuellement liée au scanner à rayons $\mathrm{X}$.

\section{Discussion}

La CIPR a publié des recommandations vis-à-vis de l'exposition aux rayonnements ionisants des femmes enceintes (ICRP, 1999). 


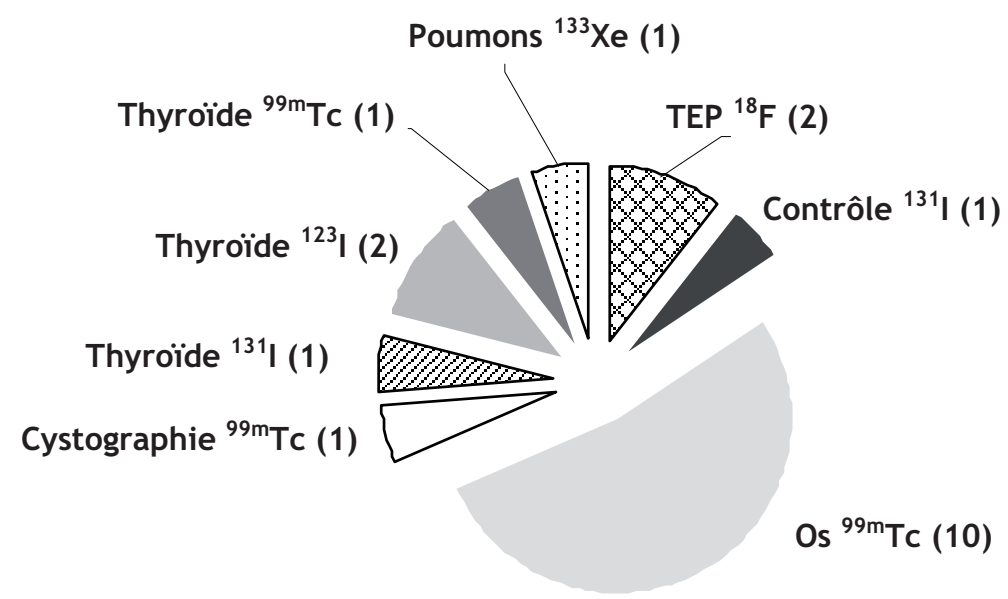

Figure 6-Répartition des types d'examen de médecine nucléaire ayant fait l'objet d'une évaluation de dose utérine.

Distribution of the assessed examinations by type, in nuclear medicine.

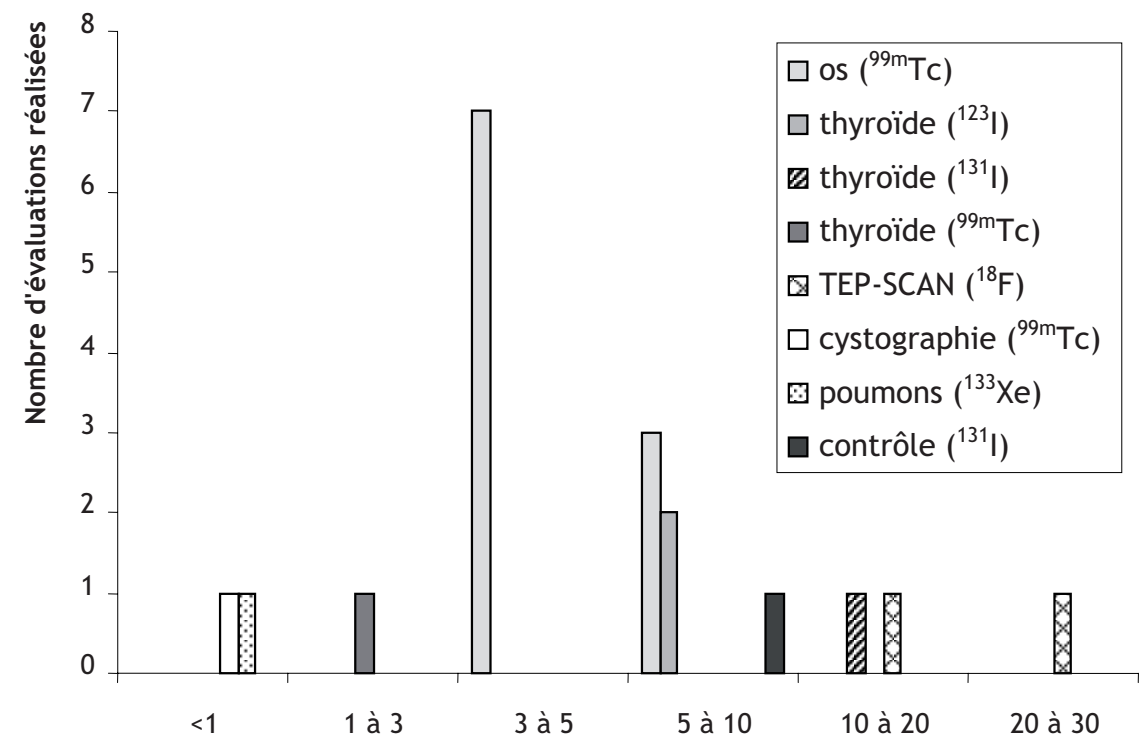

Figure 7 - Distribution des doses utérines évaluées relatives à des examens de médecine nucléaire. Distribution of the estimated foetal doses after nuclear medicine examination. 
Les principaux effets pour l'enfant à naître sont les malformations (effets déterministes) et l'induction de cancer à long terme (effets stochastiques).

Sur la base des publications internationales, la CIPR estime le seuil d'apparition des malformations autour de $200 \mathrm{mGy}$ reçus in utero. Cette même valeur seuil est retenue pour l'apparition des retards mentaux.

L'induction d'un cancer est un effet aléatoire. Son risque d'apparition augmente avec la dose, quelle que soit sa valeur. Dans l'état actuel des connaissances, le risque de cancer létal est estimé à environ $1 \%$ pour $100 \mathrm{mGy}$ reçus in utero, le risque naturel étant estimé à $0,3 \%$.

La CIPR estime que la question d'une interruption thérapeutique de grossesse sur la base du risque radique ne doit pas se poser pour une dose reçue in utero inférieure à 100 mGy.

Les valeurs de dose utérine évaluées dans cette étude selon les méthodes décrites au paragraphe 2 s'étendent de $0,2 \mathrm{mGy}$ à $200 \mathrm{mGy}$, les valeurs les plus élevées correspondant à des examens multiples (scanographie plus radiologie conventionnelle). L'incertitude associée à ces valeurs, très difficile à estimer précisément, ne saurait être inférieure à $30 \%$.

La figure 8 présente la distribution des doses évaluées par domaine.

En radiologie conventionnelle, les doses évaluées sont, à une exception près, toutes inférieures à $35 \mathrm{mGy}$, quel que soit le type d'examen. Elles sont comparables aux données de la littérature internationale.

En scanographie, les doses évaluées sont comprises dans la plupart des cas entre $15 \mathrm{mGy}$ et $65 \mathrm{mGy}$. Néanmoins, 2 examens scanographiques abdominopelviens, pour lesquels trois acquisitions ont été réalisées, ont conduit à des doses utérines supérieures à $100 \mathrm{mGy}$. Compte tenu de ces niveaux de doses et de leur dispersion, il apparaît nécessaire d'accorder une attention toute particulière à l'optimisation des examens scanographiques du pelvis chez la femme et de réaliser une évaluation individuelle de dose si la grossesse est avérée, sans se rapporter aux données de la littérature et en évitant d'utiliser une formule simplifiée.

En médecine nucléaire, la dose maximale a été évaluée à $10 \mathrm{mGy}$, suite à une scintigraphie de contrôle à l'iode 131 (200 MBq administrés). Pour les examens comportant un examen scanographique associé à la scintigraphie, la dose liée au scanner est supérieure à la dose apportée par celle-ci. Comme précisé ci-dessus, la procédure scanographique doit être optimisée au mieux. 


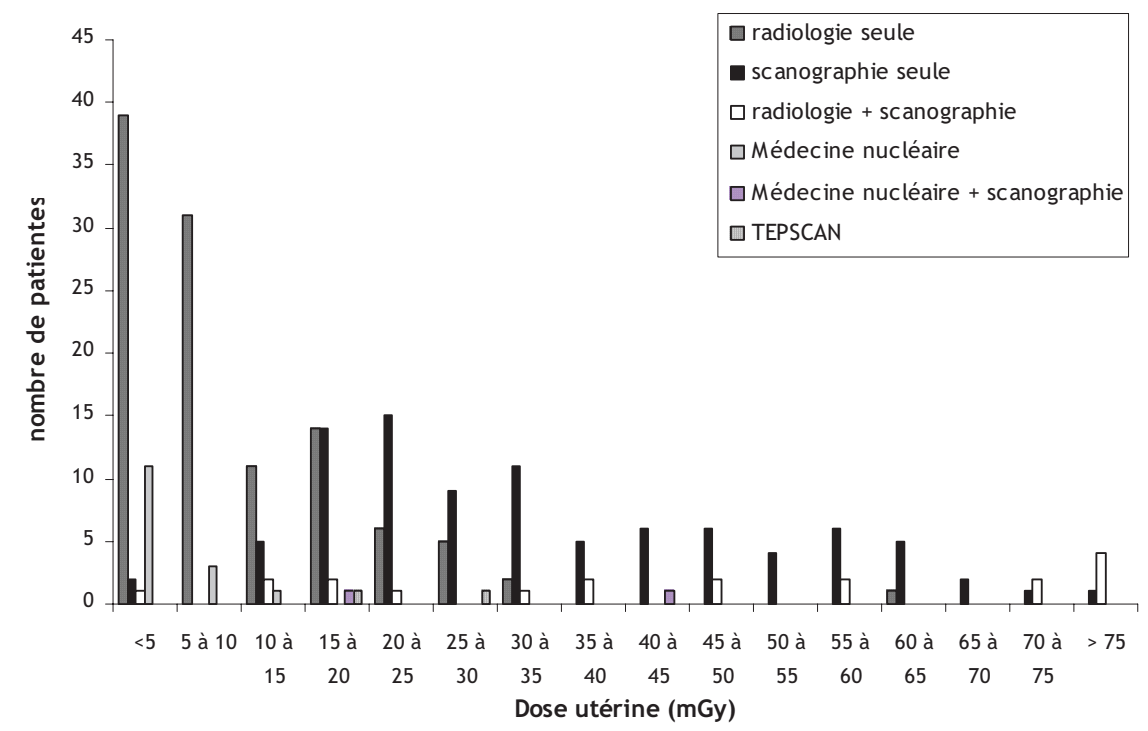

Figure 8 - Distribution des doses utérines évaluées entre 2004 et 2008, par domaine d'activité. Distribution of the estimated foetal doses according to the used image modality.

Enfin, la dose la plus élevée (200 mGy) est due à de multiples examens radiologiques et scanographiques réalisées sur une femme polytraumatisée.

\section{Conclusion}

Durant la période 2004-2008, 326 évaluations de dose utérine ont été réalisées par l'IRSN, dont 246 ont concerné des examens de radiologie et de scanographie où l'utérus se trouvait dans ou à proximité du champ exploré, ou des examens de médecine nucléaire. Parmi ces 246 cas, seules quatre patientes ont reçu des doses supérieures à $100 \mathrm{mGy}$ : deux à la suite d'un examen scanographique abdominopelvien comportant trois acquisitions sur le pelvis, et les deux autres, polytraumatisées, suite à de nombreux examens radiologiques et scanographiques.

Même si les doses utérines évaluées sont en moyenne conformes aux données de la littérature et inférieures à $100 \mathrm{mGy}$, notre étude a montré une grande dispersion des doses délivrées pour un même type d'examen. Cette dispersion observée est essentiellement liée à un non respect des recommandations des procédures de la SFR qui, sans être opposables, constituent un guide pour les professionnels permettant une homogénéisation des pratiques professionnelles. 
En scanographie, cette étude a montré que certaines configurations d'examens, ne respectant pas les recommandations de la SFR, peuvent conduire à des doses utérines jusqu'à quatre fois supérieures aux données de la littérature. Compte tenu du niveau des doses évaluées et de leur dispersion, il apparait nécessaire d'accorder une attention particulière à l'optimisation des paramètres lors des examens scanographiques du pelvis chez la femme. Une évaluation individuelle de la dose utérine reçue doit être réalisée en cas de grossesse, l'utilisation de données génériques n'étant pas suffisante.

Enfin, devant ces résultats, il convient de rappeler que les professionnels doivent systématiquement interroger leurs patientes sur une éventuelle grossesse avant chaque examen (art. R1333-61 du code de la santé publique). Si une grossesse ne peut être exclue, et si l'utérus sera dans la zone explorée, l'acte ne doit être réalisé que s'il est justifié et ne peut être retardé. Les paramètres techniques de l'examen doivent alors être optimisés pour limiter au mieux la dose délivrée.

\section{RÉFÉRENCES}

CTExpo (2007) V1.6, G.Stamm (Hannover), H.D.Nagel (Hamburg), 5th Edition, novembre 2007.

ICRP Publication 53 (1987) Radiation dose to patients from radiopharmaceuticals. Pergamon Press, Oxford.

ICRP Publication 80 (1998) Radiation dose to patients from radiopharmaceuticals. Addendum 2 to ICRP Publication 53. Pergamon Press, Oxford.

ICRP Publication 84 (1999) Grossesse et irradiation médicale. version française en 2001, EDP Sciences, Les Ulis.

IRSN (2009) IRSN/DRPH/SER n²009-2, Dose utérine en scanographie : comparaison mesures et calculs.

NRPB-R200 (1986) A national survey of doses to patients undergoing a selection of routine X-ray examinations in English hospitals. Chilton, Didcot, Oxon OX11 ORQ.

Osei E.K., Faulkner K. (1999) Fetal doses from radiological examinations, Brit. J. Rad. 72, 773-780.

PCXMC (2008) V2.0, A Monte Carlo program for calculating patient doses in medical X-ray examination, STUK, Helsinki, Finland, version 2.0, November 2008.

Sharp C. et al. (1998) Advice on exposure to ionising radiation during pregnancy. National Radiological Protection Board, Chilton, Didcot, Oxon OX11 0RQ.

SFPM (2001) n¹9-2001, Dosimétrie des explorations diagnostiques en médecine nucléaire. Société française de physique médicale. Institut Curie. Paris.

Wagner L.K. et al. (1997) Exposure of the Pregnant Patient to Diagnostic Radiations - A Guide to Medical Management. 2nd Edn. Medical Physics Publishing, Madison. 